\title{
The role of puberty and adolescence in the pathobiology of pediatric multiple sclerosis
}

Vincenzo Salpietro ${ }^{1}$, Agata Polizzi ${ }^{2,3}$, Gaia Recca ${ }^{3}$ and Martino Ruggieri ${ }^{4^{*}}$

\begin{abstract}
Multiple sclerosis (MS) is increasingly recognized in the paediatric age. In a smaller, but well-established, proportion of paediatric MS patients [ $20 \%$ of total paediatric MS cases: $0.2 \%$ to $0.7 \%$ of the total MS patients] the onset of disease is before 10 years of age [pre-pubescent (childhood) MS]; in the majority [80\%] of paediatric MS patients, however [1.7\% to $5.6 \%$ of the total MS population], the onset of disease is between 10 and 18 years [post-pubertal (juvenile) MS]. Notably, while pre-pubertal MS occurs almost equally in both genders (female/male ratio = 0.9:1; reverting to $0.4-0.6 / 1$ in pre-school MS children) the female/male ratio rises to 2.2/3:1 in the post-pubertal age. Interestingly, precocious puberty has been associated to: (a) a higher risk of developing MS; and (b) a more severe disease course. In addition to that, males are more susceptible to MS (and manifest more neurodegeneration) than females the latter being however more inflammatory than males; pregnancy however reduces MS relapses. All the above findings led to the suggestion of an underlying female sex hormonal involvement in the pathophysiology of MS vs. a protective role of male sex hormones. Epigenetic perspectives indicate that the interplay between genetic background, environmental triggers and neuroendocrine changes, typically occurring around the time of adolescence, could all play a combined role in initiating and/or promoting MS with onset in the paediatric age including many of the most frequent disease-associated risk factors (e.g., overweight/obesity, low vitamin D levels, reduced sunlight exposure, Epstein-Barr virus infection). According to this proposed complex multifactorial model, susceptibility to MS may be thus acquired during pre-pubertal age and children have probably to wait until the adolescence to manifest their first clinical signs/symptoms.
\end{abstract}

Keywords: Paediatric multiple sclerosis, Childhood multiple sclerosis, Early-onset multiple sclerosis, Puberty, Hormones, Pathophysiology, Leptin, PI3K, Demyelination

\section{Background}

The World Health Organisation (WHO) - Multiple Sclerosis International Federation reported that the interquartile range for signs/symptom onset in MS is between 25.3 and 31.8 years, placing the average age of MS onset at 29.2 years [1]. However, late-onset cases have been well documented [2] and the occurrence of MS at the other end of the spectrum of life (i.e., < age 18 years: childhood MS) is now well established (1.7\% to $10 \%$ of total MS patients) [3-19]. A small, but well-established subgroup of paediatric MS cases is younger than (or had the onset of symptoms before) 10 years of age (pre-

\footnotetext{
* Correspondence: m.ruggieri@unict.it

${ }^{4}$ Unit of Rare Diseases of the Nervous System in Childhood, Department of

Clinical and Experimental Medicine, Section of Pediatrics and Child

Neuropsychiatry, University of Catania, AOU "Policlinico-Vittorio Emanuele",

Via S. Sofia, 78, 95124 Catania, Italy

Full list of author information is available at the end of the article
}

pubescent MS: $0.2 \%$ to $0.7 \%$ of total MS cases) [7, 18] including children with onset of disease in pre-school years [17] and (exceptionally) during early infancy (i.e., < age 2 years) $[17,19]$. The mean annual incidence rates for childhood/paediatric MS is at 0.1/100,000-0.9/ 100,000 [3-16] whilst annual incidence figures for prepubescent onset MS are at 0.09/100,000] [7, 17-19]. While pre-pubescent MS occurs almost equally in both genders $($ female $/$ male ratio $=0.9: 1$; reverting to $0.4 / 0.6 / 1$ in pre-school MS children, as it occurs in acute disseminated encephalomyelitis - ADEM) [20] the female/ male ratio rises to $2.2 / 3: 1$ in the post-pubertal age (" $j u$ venile MS": i.e., MS with onset between age 10-18 years) $[7,12,17-19,21,22]$. 
In the present review, we summarize the gender effects on inflammatory and neurodegenerative processes in MS and the relationship between pubertal hormonal and/or neuroendocrine changes and the risk of paediatric MS.

\section{Pathophysiology of MS and the rationale for disease-modifying therapies}

The hallmark of MS is the demyelinated "MS plaque" that is unique and different from that seen in other inflammatory diseases and consists of a well-demarcated hypocellular area characterised by the loss of myelin, the formation of astrocytic scars, and the presence of inflammatory mononuclear cell infiltrates, typically concentrated in perivascular, particularly perivenular, cuffs [23-25]. These infiltrates, which are mainly composed of a mixture of innate (CNS-resident) and adaptive (CNS-infiltrating) components of the immune system [24], include [among the innate effectors] monocytes/macrophages, dendritic cells, reactive microglial cells, astrocytes, and mast cells, and [among the adaptive effectors] autoreactive lymphocyte $\mathrm{T}$ cells, B lymphocytes, and plasma cells plus minor additional components (e.g., ependymal cells), which after their migration into the central nervous system (CNS), incite a pro-inflammatory reaction, resulting in local tissue injury, which consists in blood brain barrier (BBB: another innate immune component) leakage, destruction of myelin sheaths, oligodendrocytes damage, and cell death, as well as axonal damage and loss, leading in turn to the glial scar (i.e., to the "MS plaque", as seen at imaging and histopathology) [23].

Thus, the migration and/or activation of (innate and adaptive) pro-inflammatory cells into the CNS represent a key stage in the natural history of MS (but what initiates this event still remains unclear) [23]. From a pathophysiologic viewpoint MS appears to be caused by a contact in early childhood with a pathogen coupled with other individual susceptibility factors (e.g., genetic, racial and demographic background), which can elicit their reactivation, triggering innate mechanisms of defence as toll-like receptors (TLRs: membrane-spanning, non-catalytic receptors expressed on sentinel cells - e.g., macrophages or dendritic cells - recognizing structurally conserved molecules derived from microbes), that signalizes downstream through its adapter protein $M y D 88$ (myeloid differentiation primary response 88 ), and the phosphorylated/degraded protein $I K B$ which permits translocation of $N F-K B$ (nuclear factor kappa-light-chain enhancer of activated $B$ cells: a protein complex, which controls DNA transcription, cytokine production and cell survival) and the transcription of pro-inflammatory cytokines such as IL-6, TNF, IL-1, IL-12, E-selectin, MCP-1, and IL-8. TLR through IRF7 (Interferon regulatory factor 7) gives the signal to the transcription of IFN $\alpha / \beta$ (i.e., the cytokines used for communication between cells to trigger the protective defences of the immune system). Another important signal is given by NOD receptors (nucleotide-binding oligomerization domain: i.e., a cytoplasmic pattern recognition receptor, which regulates the innate system and cooperates with TLRs) activated also by potassium efflux-inducing agents such as ATP and TLR stimulation. Additional signalling is provided by PAMS/PAMP (pathogen-associated molecule patterns), toxins, danger or stress, whose triggering induce the inflammasome (i.e., a cytoplasmic multiprotein oligomer) via NLRP (NOD-like receptor protein) that form a complex with ASC (apoptosis-associated speck-like protein containing a CARD: caspase recruitment domain) and caspase-1 (i.e., the interleukin-1 converting enzyme, which converts the IL precursors into mature active IL proteins), activating IL-1b, a major factor inducing inflammation, autophagy and cell death, particularly necrosis [23].

All the above pro-inflammatory soluble factors activate microglia and endothelial cells [i.e., innate effectors], upregulating expression of adhesion molecules (e.g., Eselectin), facilitating the migration of $\mathrm{T}$ cells into the CNS. Matrix metalloproteinases (MMP) degrades BBB enhancing further migration of autoreactive $\mathrm{T}$ cells and macrophages via chemokines (CX3CL-1). The Th1 response evocated via IL-12 and IFN- $\gamma$ further activates macrophages that in turn do so to T cells CD8+. Th2 response via IL-6 mainly stimulates maturation of B cells and production of autoantibodies. Cytotoxic damage to the oligodendrocyte mediates myelin loss and exposure of the axon to reactive oxygen species, slowing or blocking action potentials and the production of neurological manifestations.

There are intents to remyelinate these lesions via OPCs (oligodendrocyte precursor cells), but neuronal factors such as LINGO-1 (Leucine rich repeat and immunoglobulin-like domain-containing protein 1: a protein important for protein-protein interactions, which regulates/modulates neuronal differentiation and growth, regulation of axon guidance and regeneration processes) or TLR2 inhibit their migration [23-25].

Based on these premises, over the last two decades a dozen different preparations of immunomodulatory/immunosuppressive agents, targeting the above CNS autoimmune mechanisms, have been developed, showing beneficial effects in patients with MS and have been approved as first- or second-line disease-modifying therapies (DMTs), including [24, 26]: (a) [first-line DMTs] interferon- $\beta$ (IFN- $\beta 1 \mathrm{a}$ and $1 \mathrm{~b}$ ), glatiramer acetate (GA), dimethyl fumarate (DMF), and teriflunomide; and (b) [second-line DMTs] mitoxantrone, fingolimod (a small molecule antagonist against SIP and SIP-receptors inhibiting immune cell trafficking), natalizumab (an alpha-4 integrin blocker of immune cell trafficking/migration), alemtuzumab (an anti-CD52 cell-depleting monoclonal 
antibody), daclizumab (a blocker of the interleukin $2 \mathrm{R} \alpha$ chain), and ocrelizumab (an anti-CD20 cell-depleting monoclonal antibody) [23, 26].

Although these therapies are able to modulate the immune adaptive response, they do not inhibit innate immune cells (e.g., microglial cells, macrophages, and dendritic cells) that participate in the progression of MS. In addition to that, some of these strategies, with their indiscriminate targeting of both pathogenic and protective immune cells, might have side effects. Several new drugs are imminently emerging including strategies targeting the innate immune system [e.g., inhibition of tyrosine kinase, inhibition of NFkB, scavengers for active oxygen species and nitric oxide, or pharmacological interference with their production], or targeting the inflammasome [23].

\section{Disease-modifying therapies in pediatric MS}

No medication currently approved for adults with (relapsing-remitting) MS has completed testing for pediatric MS in randomized placebo-controlled trials, although several pediatric MS trials have recently been launched [27]. Use of DMTs in pediatric MS remains off-label in many countries, especially in patients younger than 12 years; nevertheless, these medications are widely used. At present, IFN-b and GA continue to be the standard first-line treatments for pediatric patients with MS, as supported by observational studies and experts' consensus guidelines [26, 27]. Trials are on-going evaluating the clinical outcome of pediatric patients with MS treated with fingolimod, dimethyl fumarate, and teriflunomide [27].

\section{Ages at presentation of MS in childhood and the "true" pre-pubertal threshold}

Currently, MS in the paediatric age group is divided into two main groups according to the age at presentation of first signs/symptoms [3-19]:

(1)Childhood MS (when the first acute demyelinating event occurs prior to age 12 years);

(2)Juvenile MS (when onset of disease ranges from 12 to 18 years);

A separate group defines (3) adult MS, when disease onset is after age of 18 years $[1,2]$.

The cut-off period up to 12 years to define childhood MS was chosen by most Authors in their studies because this period was (and still is) considered as the pre- or early pubertal period [Tanner stages (i.e., Breast, Genitalia, Pubic hair) I or II]. A restricted number of Authors have proposed, in their studies, a lower cut-off for defining "true" childhood MS at 10 years of age [12, 17, 19]; this (lowered) period better reflects the biological pre- pubertal period irrespective to gender [Tanner stages (i.e., Breast, Genitalia, Pubic hair) I vs. I or II: Tanner stage I represents the true pre-pubertal stage]. By lowering the cut-off period down to 10 years one could be surer: (a) to exclude early pubertal children in analysis of paediatric MS cases, thus avoiding inclusion of MS patients already targeted by the postulated effects of pubertal sex hormones on predisposed tissues [e.g., bone marrow, thymus, central nervous system]; and (b) to limit multiple viral exposures as by age 10 years most children (e.g., in Italy) have usually completed their vaccination schedule of mandatory and recommended vaccines $[17,19]$.

Pre-pubescent onset MS is characterised by peculiar clinical, laboratory and imaging features and outcome $[17,19,28]$, including inversion of sex ratios, low to null family history for MS, preponderance of atypical manifestations at onset (e.g., hemiparesis, seizures, lethargy, brainstem signs/symptoms or cerebellar ataxia), polyfocal presentation, highest relapse number/year and fastest recovery time, more severe neurological deficits at relapses with more completely or near-completely recovery, ADEM/leukodystrophy-like MRI patterns at onset vs. typical MS MRI patterns attained years after the first attacks, a worse outcome in the earliest onsets (i.e., < 2 years of age) vs. a better outcome (as compared to post-pubertal MS) in onsets at toddler ages.

\section{Age- and gender-related peculiarities of pediatric MS vs. similar disorders}

A peculiar female responsiveness to environmental triggers is noted across many disease models and is usually attributed to the need, in the female gender, to make repeated, rapid and consistent physiologic accommodations to pregnancy. In female adolescents with MS, a number of genetic, non-genetic and lifestyle factors have possibly sexually dimorphic effects on MS disease predisposition and on its clinical course [29,30].

Similarly to what occurs in MS, the so-called pseudotumour cerebri syndrome (PTCS) is a neurological disorder, which, within childhood, mostly affects post-pubertal females, who often are overweight. PTCS is a condition of unclear aetiology, characterised by increased intracranial pressure (ICP) without any radiographic evidence of brain tissue abnormalities, and with normal chemical and cytological cerebrospinal fluid (CSF) composition [31-33]. Multiple causes have been taken into consideration in the pathophysiology and aetiology of PTCS [32, 33] including obesity, endocrine abnormalities (e.g., hyperaldosteronism, Cushing syndrome, hyperandrogenism, Addison disease), kidney disease (e.g., nephrotic syndrome), systemic disease (e.g., systemic lupus erythematous, Guillain-Barrè syndrome, antiphospholipid antibody syndrome, polycystic ovary syndrome - PCOS, Behcet disease, familial 
Mediterranean fever), medications (e.g., recombinant growth hormone therapy, tetracycline, steroids, mycophenolate mofetil, vitamins A and D, cytarabine, and cyclosporine A), viral infections (e.g., chickenpox, measles, reactivation of varicella infection) and changes in CSF volume and in cerebral CSF hemodynamic (increased cerebral blood volume, increased cerebrospinal fluid production, decreased cerebrospinal fluid resorption or venous flow abnormalities); PTCS has been also observed in members of the same family presenting in either an autosomal dominant or recessive manner. A recently proposed unifying (neuroendocrine) hypothesis inferred that [32] multiple neuroendocrine interactions (e.g., cortisol, aldosterone, progesteron) could influence the activation of the mineralocorticoid receptor (MR) in the choroid plexus epithelial cells, which in turn stimulates (via a nuclear pathway) the ATPase $/ \mathrm{Na}^{+} / \mathrm{K}^{+}$pump leading to raised intracranial CSF production [25]. Even though it typically affects both genders and all age groups, the post-pubertal PTCS typically occurs in overweight girls/women during their reproductive age [34]. Notably, the overall incidence of PTCS is estimated to be $0,9 / 100,000$ rising to $19 /$ 100,000 in overweight women $[34,35]$.

Paediatric PTCS is known to occur in association with a broad variety of conditions, especially obesity and endocrine derangements (e.g., cortisol deficiency or excess, hyperandrogenism, hyperaldosteronism) [32, 36]. Although pre-pubertal PTCS can occur in both genders and ages, post-pubertal PTCS is usually recorded in women during their reproductive age $[34,35]$ : in this respect, it has been previously proposed that the proneness of some women to develop PTCS could be linked to an estrogenic gynecoid (pear-shaped) fat distribution [34]. Adipose tissue contains aromatase, which may be a link between obesity and PTCS. Aromatase, which catalyses the production of oestrogens from plasma androstenedione, is more prevalent in the fat of the buttock regions (reflecting the typical female fat distribution) vs. the abdominal (visceral) regions [34-36]. Of note, the reports of the onset of PTCS in postmenopausal women following the initiation of hormone replacement therapy further support the notion of an oestrogen involvement in the pathophysiology of this condition [31-33, 37, 38].

The PTCS neuroendocrine pathophysiology [32] cannot be applied to MS, as the mechanism underlying the rise of CSF pressure cannot be compared to the process of demyelination and unlikely involves an autoimmune aetiology [39]. Nonetheless, higher values of ICP have been recently documented in the paediatric MS population [31, 32], thus reflecting the fact that both these conditions (PTCS and MS) could share similar precipitating factors (e.g., obesity, female sex hormones) on a background of alike clinical and anthropometric features. A tenable hypothesis of common possible trigger(s) underlying both diseases could be represented by the putative involvement of Leptin, which seem to be centrally involved either in PTCS and in MS pathophysiology [7, 32] (Fig. 1).

\section{The role of gender factors in paediatric MS}

The sex discrepancy (with a female preponderance) in MS is evident only in individuals who manifest disease symptoms after puberty $[1,7,17,19]$, implicating a likely role of female sex hormones in initiating and/or promoting the disease [7] and of post-pubertal male (high) levels of testosterone in protecting from the disease [40]. The above notion is supported by a number of clinical and laboratory evidences: (a) men with MS present at an older age, concurrent with the start of the age-related decline in testosterone levels; (b) a decrease of androgen levels in MS adult males is associated with a more severe disease course and a faster progression to disability; and (c) testosterone administration may ameliorate the clinical course of MS in males [41, 42].

Oestrogens (17 $\beta$-estradiol-E2- and estriol-E3), progesterone and testosterone may provide anti-inflammatory and neuroprotective effects on induction and effector phases of experimental allergic encephalomyelitis (EAE) $[29,30]$. Anti-inflammatory effects appear mainly mediated by oestrogen nuclear receptors alpha (ER $\alpha)$ and beta (ER $\beta)$ expressed by regulatory CD $4+C D 25+T$ cells (Treg), regulatory B (Breg) cells and dendritic cells and may be abrogated in the absence of $\mathrm{B}$ cells and the coinhibitory receptor, Programmed Death-1 (PD-1) on CD4+ Foxp3+ Treg cells. E2 protective effects on EAE seem to be mediated by binding to the membrane Gprotein-coupled receptor 30(GPR30). Testosterone may work through androgen receptors or after its conversion to oestrogen through ERs, or GPR30. Androgens may induce remyelination in cuprizone-induced CNS demyelination by acting on neural androgen receptors. Experimental studies also showed that androgens exert a protective role against the development of EAE, the animal model of MS [30]. Additionally, therapeutic trials with dihydrotestosterone (DHT) in castrated animals ameliorate both symptoms and inflammation [29, 30, 40].

Some neuroprotective effects of oestrogens in EAE are mediated by ER $\alpha$ expressed on astrocytes: ER $\beta$ ligands can prevent demyelination and stimulate remyelination and ER $\beta$ treatment can affect microglia with protective effects in CNS inflammation. Progesterone appears to affect axonal protection and remyelination, and testosterone can restore synaptic transmission deficits in the hippocampus.

Sex hormones play a pivotal role in the human immune system, regulating antigen presentation, cytokine gene expression, lymphocyte activation and autoimmune 


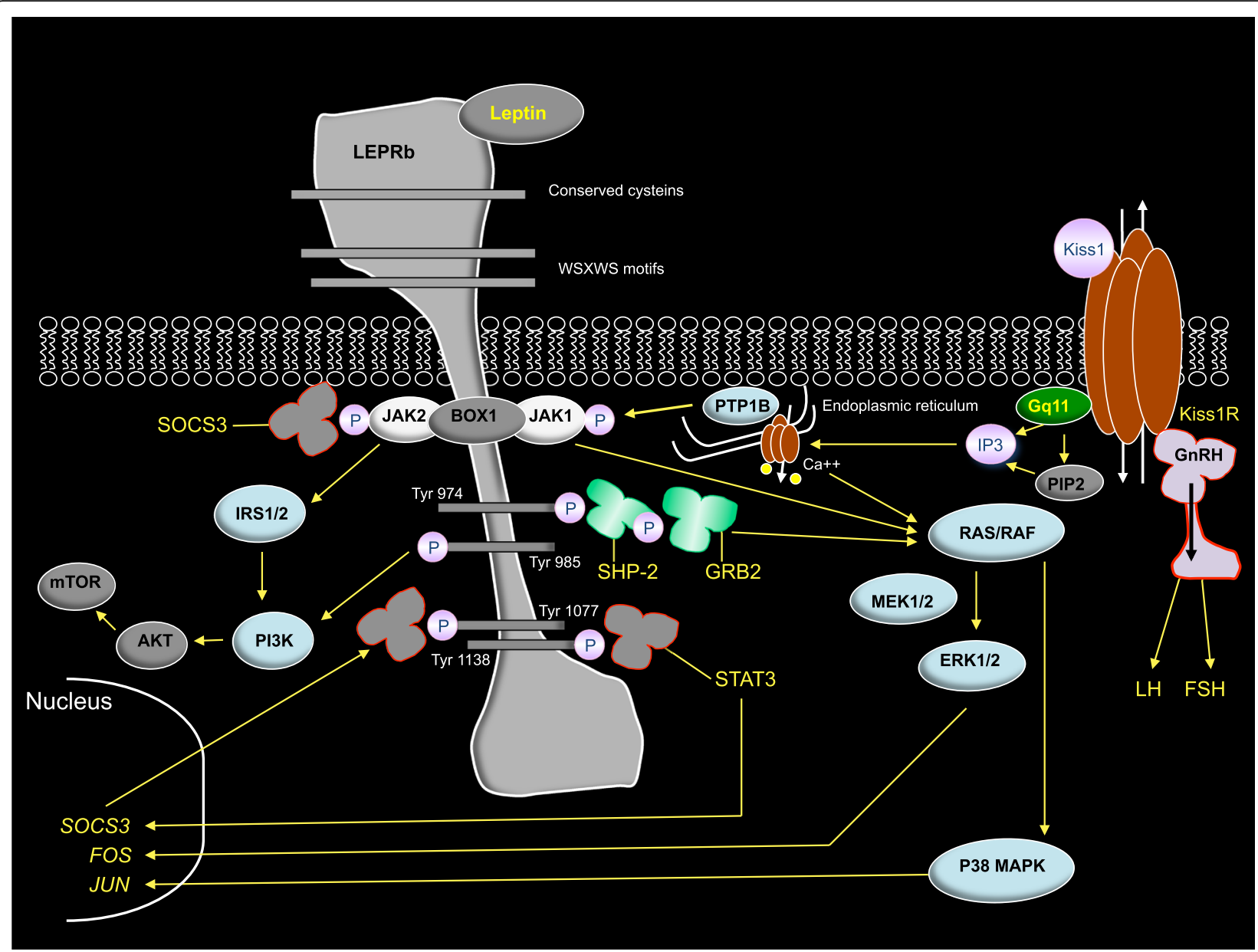

Fig. 1 Mechanisms of Leptin signalling in immune and neuroendocrine cells. Leptin binds to one of its receptors, LEPRb, activating JAK2 by autophosphorylation or cross-phosphorylation, and phosphorylates tyrosine residues in the receptor's cytoplasmic domain. Four of the phosphorylated residues $[974,985,1077,1138]$ function as docking sites for cytoplasmic adaptors for STAT factors, particularly STAT3, which dimerizes translocating into the nucleus, where it induces expression of SOCS3, FOS and JUN genes. SOCS3 participates in a feedback loop that inhibits Leptin signalling by binding to phosphorylated tyrosines. SHP-2 is recruited to Tyr985 and Tyr974 and activates ERK1/2 and P38 MAPK pathways through the adaptor protein GRB2, ultimately inducing FOS and JUN gene expression [FOS and JUN encode for fos and jun proto-oncogene proteins, which form heterodimers (C-fos:C-jun) resulting in the formation of AP-1 (Activator Protein-1) complex, which binds DNA at AP-1 specific sites at the promoter and enhancer regions of target genes and converts extracellular signals into changes of gene expression]. PTP-1B is localized on the surface of the endoplasmic reticulum, and is involved in negative regulation of LEPRb signalling through dephosphorylation of JAK2 after internalization of the LEPRB complex; the endoplasmic reticulum is also the site of action (via Ca++) of the IP3-PIP2-mediated pathway of the Kissprotein 1, which in turn modulates GnRH secretion and ultimately LH and FSH secretion [neuroendocrine cells are hereby represented as if they were inside the membrane for practical purposes: in the real pathways the Kiss1 protein binds to the Kiss1 receptor (R), which is expressed on the membrane surface of both immune and neuroendocrine cells: the latter cells promote secretion of GnRH, which in turn stimulate secretion of LH and FSH]. JAK2 can also induce phosphorylation of the IRS1 and 2 proteins, which are responsible for PI3K/AKT and mTOR pathway activation

processes [30, 41]. Also, immune central tolerance at the thymus level is strictly dependent on the hormonal status [29, 30, 42]. Elevation of sex steroids during puberty has been, de facto, linked to the typical decline of the thymus, which starts around adolescence; the thymus rejuvenation after ablation of sex steroids further supports this notion [29, 30, 43]. It is unsure whether puberty and its related hormonal changes affect the susceptibility to environmental factors such as infections.
There are gender-related differences in immune response and women have higher levels of immunoglobulin and more vigorous T-cell activation when compared to males [44]. Oestrogens appear to have a controversial role on inflammation in EAE. At lower levels, oestrogens - such as estradiol - may promote inflammation; but at higher levels, oestrogens - such as the pregnancy hormone estriol - may induce a shift in the immune response from a $\mathrm{T}$ helper 1 (TH1) response to a $\mathrm{T}$ helper 2 (TH2) response, muting inflammation [45]. This 
would explain the reason for which disease activity usually decreases during late pregnancy, which is typically characterized by high levels of estriol and also the beneficial effects of estriol administration to nonpregnant MS females in improving the disease manifestations [46-48].

Studies in EAE also show that low dose oestrogen therapy may have also profound effects in inhibiting the development of autoimmunity, likely influencing the immune reaction towards a protective antiinflammatory cytokine response [29, 30]. However, in one of these studies, oestrogen treatment at the onset of active EAE failed to reduce disease severity, a result that is consistent with the hypothesis that naive cells are more sensitive to sex hormones than differentiated effector cells [49].

Of note, post-pubertal EAE female mice develop increased myelin reactive T-cell responses compared to agematched mice that had been prevented from entering puberty via pre-pubertal ovariectomy surgery $[49,50]$. Together, these studies suggest that puberty in females enhances central nervous system (CNS) autoimmune mechanisms, further explaining the female preponderance of MS, at the post-pubertal ages.

Lastly, the role of the female chromosome $\mathrm{X}$ on immunity and MS should be also regarded as crucial. This could involve hormones-independent mechanisms, including microRNAs and cytokine genes present on chromosome X [51].

\section{Precocious puberty and the risk of MS}

Recent MS studies, further deepened, the (causal) relationship between puberty and the disease: initially, only the peri-pubertal period was regarded as typically associated to a dramatic, female-specific, rise in disease incidence; later studies, however, demonstrated that an earlier occurrence of puberty and menarche was also associated to higher risks of disease onset and a more severe clinical course. One study demonstrated that the age at first symptoms increased by 1.16 years as the age of menarche increased by one year [52]. A further MS collaborative Canadian study showed that females with MS were younger at menarche (i.e., 12.4/12 years vs. 12.6/12 years) compared to controls [53]. An association between earlier age at menarche in females and a more severe disease course has been also recorded [54].

A potential effect of age of puberty and menarche on MS, further strengthens the putative involvement of female sex hormones in disease pathophysiology, due to oestrogen-related changes in CNS and immune system (as outlined above). Thus, an earlier menarche may possibly upset a delicate oestrogen balance, making some susceptible girls prone to develop MS.
However, the question whether younger age at puberty is a real trigger for the disease or a mere trigger factor on a background of multiple genetic and environmental determinants remains unsolved. Additionally, it has also been speculated that earlier menarche is a surrogate for the effect of an MS disease causative factor that influences the risk of MS independently by oestrogens, whilst affecting the age of menarche as a by-product [53].

Of note, puberty onset requires specific changes in the secretion of the pituitary gonadotropins, luteinizing hormone (LH) and follicle-stimulating hormone (FSH), which are dependent on the release of Gonadotropin [LH/ FSH] releasing hormone (GnRH) from the hypothalamus (Fig. 1); thus, timing of puberty is strictly dependent on a specific genetic susceptibility and on environmental conditions that can influence physiological and pathological processes acting on the hypothalamic-pituitary axis, including nutrition, adiposity, bone mass, emotional and psychological factors, light-darkness cycles/melatonin and endocrine disrupting compounds [55].

Interestingly, also low vitamin D levels are usually associated with an earlier age of pubertal onset in the paediatric population [56]. In this context, it is still unclear whether environmental exposures affecting puberty timing also affect the risk of developing MS in an independent manner. Thus, the correlation between precocious timing of puberty and menarche and the risk of onset and/or worsening of MS and the higher prevalence of obesity within the paediatric MS population are overall factors, which reflect a possible underlying endocrinology/metabolic involvement in the pathophysiology of MS.

Some important MS-associated risk factors (i.e., low vitamin D level, obesity) are also known to be causes of early puberty per se, further supporting the possibility that earlier puberty is a surrogate for the effect of an MS disease causative factor that influences both the risk of MS and an earlier menarche.

\section{Obesity and Leptin: Correlations between metabolism and paediatric MS}

Pediatric obesity has been demonstrated in one study to be a risk factor for later development of adult-onset MS in women whilst obesity occurring in adulthood carried out a null risk of developing MS [57]. Another study found that paediatric obesity was independently associated with an increased risk of paediatric onset MS in girls but not in boys: the association between body mass index (BMI) and paediatric MS was strikingly pronounced in extremely obese adolescent girls [7].

Despite these findings, still there are many underexplored and/or not yet fully understood aspects on these relationships. The relative percentages of body fat during the paediatric age are known to be associated with 
accelerated sexual maturation and precocious puberty: it is unclear if overweight and obesity may predispose independently to both earlier puberty and MS, or if it happens in a consecutive manner [58, 59]. Obesity is characterized by a low-grade inflammation state and it is known to be associated with a T helper 17 (Th17) bias predisposing to autoimmune reactions [60]. Additionally, interactions between obesity and vitamin D status remains unexplored [47].

Adipose tissue is not an inert tissue implied only in energy storage, but can be regarded as a part of an endocrine organ, which releases many mediators that in turn may predispose to both puberty and MS; additionally, some of these mediators and/or adipokines released by adipocytes are involved in several inflammatory processes, including tumor necrosis factor alpha (TNF-a), interleukin 6 (IL-6) and Leptin. The adipokine hormone Leptin is an amino-acid cytokine-like protein, which is known to play a crucial role in regulating puberty, especially in females. At central (hypothalamic) level, Leptin facilitates puberty onset likely stimulating the Kisspeptin1 (Kiss1) pathways, the upstream regulators of $\mathrm{GnRH}$ neurons [55] (Fig. 1).

Besides its metabolic role in promoting puberty onset, Leptin has many additional central and/or peripheral actions, including regulation of both innate and adaptive immunity. In fact Leptin stimulates the secretion of pro-inflammatory cytokines (e.g., Il-6, IL-18) and at the adaptive immune system level, Leptin promotes switch towards pro-inflammatory Th1 immunological responses [61].

Leptin mediates its effects by binding to Leptin receptors (LepRs) expressed in the brain and a in wide array of peripheral tissues. Various alternatively spliced isoforms of LepRs have been described, but the long isoform of Leptin receptor (LepRb) is primarily responsible for Leptin signalling (Fig. 1). The binding of Leptin to LepRb activates a number of signalling pathways, including AK2/STAT 3 and STAT5, SHP2/MAPK and PI3K/AKT/mTOR [59-61]. Notably, the activation of the phosphatidylinositol-3 kinase (PI3K) pathway by Leptin is one of the most studied effects of Leptin signalling in the brain and it has been demonstrated to play crucial roles in several metabolic and energetic processes [61]. A number of studies have demonstrated the relevance of PI3K as an underlying mechanism of Leptin actions in vivo [52]. In rats, peripheral Leptin administration was found to activate PI3K in the brain and pre-treatment with inhibitors of PI3K abolished the anorectic response induced by Leptin [61-63].

Interestingly, the balance of PI3K/AKT pathway is essential for oligodendrocytes survival and axon myelination and gain of functions mutations of genes enclosed in this pathway (especially mutations in PIK3CA gene) have been linked to various types of overgrowth syndromes [known as PIK3-related overgrowth syndromes, or PROS] [64, 65], which are also characterized by diffuse white matter abnormalities and increased signal on T2-weighted images on MRI [66, 67]. Studies that investigate potential interactions between Leptin and the PI3K signalling in MS patients are needed.

One of the principal observations, which indicate that Leptin could represent a key mediator in the pathogenesis of MS, is due to its female-specific rise during the peri-pubertal age. In fact, numerous studies showed that during pubertal age Leptin levels continue to increase in girls but not in boys due to the testosterone-related inhibition on Leptin secretion [63]. Besides its interaction with the PIK/AKT pathway, possibly implicated in early cytodegenerative processes of myelin, the actions of Leptin include a strong influence in both the innate and the adaptive immune system. In the innate system, Leptin stimulate the activation of the monocyte-macrophage lineage and the secretion of pro-inflammatory cytokines; in the adaptive immune system Leptin induces proinflammatory Th1 responses [61, 62]. Interestingly, the Leptin deficient mice are resistant to the induction of EAE, and administration of Leptin in this animal model shifts the Th2-type response, characteristic of this animal model, to a Th1-type response [68].

Moreover, it has been observed that Leptin is able to maintain environmental conditions that promote loss of immune self-tolerance $[69,70]$; in particular, both in vitro and in vivo, leptin can affect the generation, proliferation and responsiveness of $t_{r e G}$ cells, a key type of $t$ cells that is involved in the control of immunological tolerance [71].

Thus, the crucial involvement of leptin in initiating and promoting puberty, the observation that it continues to rise in female but not in male adolescents, the increased levels of leptin in the pediatric obese population, the central role of this hormone in regulating inflammatory and autoimmune processes, the demonstration of its necessary role for the induction of the animal model of MS (i.e., EAE), are all convincing evidences of the involvement of leptin in pathogenesis of MS, especially in the post-pubertal pediatric age group.

\section{Conclusions and future directions}

In conclusion, within the post-pubertal MS group both the disease prevalence and the female-male ratio are much higher if compared to the pre-pubertal MS group. Furthermore, a more precocious onset of puberty has been associated to both a higher risk of developing MS and an even more severe disease course. Additional support linking puberty with the pathogenesis of MS may be driven from the observations of remarkable similarities between the neuroendocrine mechanisms underlying the onset of puberty and those associated with the 
postpartum period [72]. In fact, the hypogonadotrophic state of the postpartum phase resembles the prepubertal (hypogonadotrophic) state [73, 74].

Interestingly, the postpartum recovery of gonadotropin release follows a predictable sequence of a preferential rise of FSH followed by LH secretion, a pattern identical to that of the peri-pubertal state [72]. For these reasons, the neuroendocrine changes in the postpartum period have been also known as "puberty in miniature" and have been frequently associated to the significantly increased risk of onset and relapse of MS after partum. Thus, it is possible that the biological mechanisms, which are responsible for the development of the clinical manifestations of MS in the pubertal or post-pubertal periods, are also involved in the onset or the reactivation of the disease in the post-partum period.

Thus, recent researches in the field suggest that the neuroendocrine changes, typically occurring around the time of puberty, could play a role in initiating and/or promoting pediatric MS associated with additional genetic/non-genetic (e.g., environmental) factors.

According to this multifactorial model, susceptibility to MS may be thus acquired during a wide window of risk through childhood and most pre-pubertal children acquiring susceptibility to the disease have probably to wait until the "pubertal switch" to manifest the clinical symptoms. Further experimental studies are required in future to fully understand the gene-neuroendocrineimmune-environment-lifestyle interactions underlying the molecular pathobiology of pediatric-onset MS.

\begin{abstract}
Abbreviations
AKT: AK (Akr mouse) strain transforming; FOS: Finkel osteogenic sarcoma [Finkel-Biskis-Jinkins murine osteogenic sarcoma virus]; FSH: Follicle stimulating hormone; GnRH: Gonadotropin [LH/FSH] releasing hormone; Gq11: Guanine nucleotide binding protein q 11; GRB2: Growth factor receptor-bound protein 2; IRS: Insulin receptor substrate; JAK2: Janus kinase 2; JUN: Jinkins avian sarcoma virus oncogene; Kiss1: Kissprotein 1; Kiss1R: Kissprotein receptor; LEPRb: Leptin receptor, long form b; LH: Luteinizing hormone; MAPK: Mitogen-activated protein kinase; MEK2: MAPK-extracellular kinase 2; mTOR: Mammalian target of rapamycin; PI3K: Phosphatidylinositol-3-kinase; PIP3: Phosphatidylinositol tri-phosphate; PTP-1B: Tyrosine-protein phosphatase non-receptor type 1; RAF: Rapidly accelerated fibrosarcoma protein; RAS: Rat sarcoma viral (V-ras) oncogene homolog; SHP-2: SHP protein tyrosine phosphatase-2; SOCS3: suppressor of cytokine signalling 3; STAT3: transducer and activator of transcription 3; IP3: Inositol 1,4,5-trisphosphate receptor, type 3; ERK1/2: Extracellular-signal regulated kinases 1 and 2
\end{abstract}

\section{Acknowledgements}

We wish to thank Dr. G.H. Tutino (Catania) for his valuable comments and support.

\section{Funding}

None

\section{Availability of data and materials}

Not applicable

\section{Authors' contributions}

VS and MR conceived the review, participated in its design and coordination and wrote the initial draft. VS and GR reviewed the existing literature. VS and
AP drafted the final version. AP along with MR designed and drew Fig. 1. All authors read and approved the final manuscript.

Ethics approval and consent to participate

This was submitted to and approved by the Ethical Committee (Catania 1) based at the AOU "Policlinico-Vittorio Emanuele", Catania.

\section{Consent for publication}

All Authors were informed and gave their consent to publication in MSDD.

Competing interests

The authors declare that they have no competing interests.

\section{Publisher's Note}

Springer Nature remains neutral with regard to jurisdictional claims in published maps and institutional affiliations.

\section{Author details}

${ }^{1}$ Department of Molecular Neuroscience, University College of London, London, UK. ${ }^{2}$ National Centre for Rare Diseases, Istituto Superiore di Sanità, Rome, Italy. ${ }^{3}$ Institute of Neurological Sciences, National Research Council, Catania, Italy. ${ }^{4}$ Unit of Rare Diseases of the Nervous System in Childhood, Department of Clinical and Experimental Medicine, Section of Pediatrics and Child Neuropsychiatry, University of Catania, AOU "Policlinico-Vittorio

Emanuele", Via S. Sofia, 78, 95124 Catania, Italy.

Received: 2 March 2017 Accepted: 10 November 2017

Published online: 22 February 2018

\section{References}

1. Compston A, Coles A. Multiple sclerosis. Lancet. 2008;372:1502-17.

2. Kis B, Rumberg B, Berlit P. Clinical characteristics of patients with late-onset multiple sclerosis. J Neurol. 2008;255:697-02.

3. Duquette P, Murray TJ, Pleines J, Ebers GC, Sadovnick D, Weldon P, Warren S, Paty DW, Upton A, Hader W, et al. Multiple sclerosis in childhood: clinical profile in 125 patients. J Pediatr. 1987;111:359-63.

4. Ghezzi A, Deplano V, Faroni J, Grasso MG, Liguori M, Marrosu G, Pozzilli C, Simone IL, Zaffaroni M. Multiple sclerosis in childhood: clinical features of 149 cases. Mult Scler. 1997;3:43-6.

5. Mikaeloff Y, Caridade G, Assi S, Suissa S, Tardieu M. Prognostic factors for early severity in a childhood multiple sclerosis cohort. Pediatrics. 2006;1 18:1133-9.

6. Renoux C, Vukusic S, Mikaeloff Y, Edan G, Clanet M, Dubois B, Debouverie M, Brochet B, Lebrun-Frenay C, Pelletier J, Moreau T, Lubetzki C, Vermersch P, Roullet E, Magy L, Tardieu M, Suissa S, Confavreux C, Adult Neurology Departments KIDMUS Study Group. Natural history of multiple sclerosis with childhood onset. N Engl J Med. 2007;356:2603-13.

7. Banwell B, Ghezzi A, Bar-Or A, Mikaeloff Y, Tardieu M. Multiple sclerosis in children: clinical diagnosis, therapeutic strategies, and future directions. Lancet Neurol. 2007;6:887-902.

8. Krupp LB, Tardieu M, Amato MP, Banwell B, Chitnis T, Dale RC, Ghezzi A, Hintzen R, Kornberg A, Pohl D, Rostasy K, Tenembaum S, Wassmer E, International Pediatric Multiple Sclerosis Study Group. International pediatric multiple sclerosis study group criteria for pediatric multiple sclerosis and immune-mediated central nervous system demyelinating disorders: revisions to the 2007 definitions. Mult Scler. 2013;19:1261-7.

9. Pohl D, Hennemuth I, von Kries R, Hanefeld F. Paediatric multiple sclerosis and acute disseminated encephalomyelitis in Germany: results of a nationwide survey. Eur J Pediatr. 2007;166:405-12.

10. Chitnis T, Glanz B, Jaffin S, Healy B. Demographics of pediatric-onset multiple sclerosis in an MS center population from the northeastern United States. Mult Scler. 2009;15:627-31

11. Simone IL, Carrara D, Tortorella C, Liguori M, Lepore V, Pellegrini F, Bellacosa A, Ceccarelli A, Pavone I, Livrea P. Course and prognosis in early-onset MS: comparison with adult-onset forms. Neurology. 2002;59:1922-8.

12. Achiron A, Garty BZ, Menascu S, Magalashvili D, Dolev M, Ben-Zeev B, Pinhas-Hamiel O. Multiple sclerosis in Israeli children: incidence, an clinical, cerebrospinal fluid and magnetic resonance imaging findings. Isr Med Assoc J. 2012;14:234-9.

13. Reinhardt K, Weiss S, Rosenbauer J, Gartner J, von Kries R. Multiple sclerosis in children and adolescents: incidence and clinical picture - new insights 
from the nationwide german surveillance (2009-2011). Eur J Neurol. 2014;21:654-9.

14. Ghezzi A, Ruggieri M, Trojano M, Filippi M, ITEMS Study Group. Italian studies on early onset multiple sclerosis: the present and the future. Neurol Sci. 2004;25(supp|4):S346-9.

15. Van Haren $\mathrm{K}$, Waubant E. Therapeutic advances in paediatric multiple sclerosis. J Pediatr. 2013;163:631-7.

16. Chitnis T, Tardieu M, Amato MP, Banwell B, Bar-Or A, Ghezzi A, Kornberg A, Krupp LB, Pohl D, Rostasy K, Tenembaum S, Waubant E, Wassmer E. International pediatric MS study group clinical trials summit: meeting report. Neurology. 2013;80:1161-8

17. Ruggieri M, Polizzi A, Pavone L, Grimaldi LME. Multiple sclerosis in children under 6 years of age. Neurology. 1999;53:478-84.

18. Huppke B, Ellenberger D, Rosewich H, Friede T, Gärtner J, Huppke P. Clinical presentation of pediatric multiple sclerosis before puberty. Eur J Neurol. 2014;21:441-6

19. Ruggieri M, lannetti P, Polizzi A, Pavone L, Grimaldi LM, Italian Society of Paediatric Neurology Study Group on Childhood Multiple Sclerosis. Multiple sclerosis in children under 10 years of age. Neurol Sci. 2004;25(Suppl 4):S326-35.

20. Pavone P, Pettoello-Mantovano M, Le Pira A, Polizzi A, Giardino I, Parano E, Pulvirenti A, Giugno R, Ferro A, Pavone L, Ruggieri M. Acute disseminated encephalomyelitis. A long-term prospective study and meta-analysis of the literature. Neuropediatrics. 2010;41:246-55.

21. Orton SM, Herrera BM, Yee IM, Valdar W, Ramagopalan SV, Sadovnick AD, Ebers GC, Canadian Collaborative Study Group. Sex ratio of multiple sclerosis in Canada: a longitudinal study. Lancet Neurol. 2006;5:932-6.

22. O'Connor KC, Lopez-Amaya C, Gagne D, Lovato L, Moore-Odom NH, Kennedy J, Krupp L, Tenembaum S, Ness J, Belman A, Boyko A, Bykova O, Mah JK, Stoian CA, Waubant E, Kremenchutzky M, Ruggieri M, Bardini MR, Rensel M, Hahn J, Weinstock-Guttman B, Yeh EA, Farrell K, Freedman MS, livanainen M, Bhan V, Dilenge M, Hancock MA, Gano D, Fattahie R, Kopel L, Fournier AE, Moscarello M, Banwell B, Bar-Or A. Anti-myelin antibodies modulate clinical expression of childhood multiple sclerosis. J Neuroimmunol. 2010;223:92-9.

23. Hernández-Pedro NY, Espinosa-Ramirez G, de la Cruz VP, Pineda B, Sotelo J. Initial immunopathogenesis of multiple sclerosis: innate immune response. Clin Dev Immunol. 2013:2013:413465.

24. Waisman A, Liblau RS, Becher B. Innate and adaptive immune response in the CNS. Lancet Neurol. 2015;14:945-55.

25. Koudriavtseva T, Mainero C. Neuroinflammation, neurodegeneration and regeneration in multiple sclerosis: intercorrelated manifestations of the immune response. Neural Regen Res. 2016;11:1727-30.

26. Ingwersen J, Aktas $\mathrm{O}$, Hartung HP. Advances in and algorithms for the treatment of relapsing-remitting multiple sclerosis. Neurotherapeutics. 2016;13:47-57.

27. Ghezzi A, Amato MP, Makhani N, Shreiner T, Gärtner J, Tenembaum S. Pediatric multiple sclerosis: conventional first-line treatment and general management. Neurology. 2016;87(9 Suppl 2):S97-S102.

28. Waldman A, Ness J, Pohl D, Simone IL, Anlar B, Amato MP, Ghezzi A. Pediatric multiple sclerosis: clinical features and outcome. Neurology. 2016:87(9 Suppl 2):S74-81.

29. Miller DH, Fazekas F, Montalban X, Reingold SC, Trojano M. Pregnancy, sex and hormonal factors in multiple sclerosis. Mult Scler. 2014;20:527-36.

30. Ramien C, Taenzer A, Lupu A, Heckmann N, Engler JB, Patas K, Friese MA, Gold SM. Sex effects on inflammatory and neurodegenerative processes in multiple sclerosis. Neurosci Biobehav Rev. 2016;67:137-46.

31. Soiberman U, Stolovitch C, Balcer L, Regenbogen M, Constantini S, Kesler A. Idiopathic intracranial hypertension in children: visual outcome and risk of recurrence. Childs Nerv Syst. 2011;27:1913-8.

32. Salpietro V, Polizzi A, Bertè LF, Chimenz R, Chirico V, Manti S, Ferraù V, Salpietro A, Arrigo T, Ruggieri M. Idiopathic intracranial hypertension: a unifying neuroendocrine hypothesis through the adrenal-brain axis. Neuro Endocrinol Lett. 2012;33:569-73.

33. Salpietro V, Mankad K, Kinali M, Adams A, Valenzise M, Tortorella G, Gitto E, Polizzi A, Chirico V, Nicita F, David E, Romeo AC, Squeri CA, Savasta S, Marseglia GL, Arrigo T, Johanson CE, Ruggieri M. Pediatric idiopathic intracranial hypertension and the underlying endocrine-metabolic dysfunction: a pilot study. J Pediatr Endocrinol Metab. 2014;27:107-15.

34. Andrews LE, Liu GT, Ko MW. Idiopathic intracranial hypertension and obesity. Horm Res Paediatr. 2014;81:217-25.

35. Radhakrishnan K, Ahlskog JE, Cross SA, Kurland LT, O'Fallon WM. Idiopathic intracranial hypertension (pseudotumor cerebri). Descriptive epidemiology in Rochester, Minn, 1976 to 1990. Arch Neurol. 1993;50:78-80.
36. Sheldon CA, Kwon YJ, Liu GT, McCormack SE. An integrated mechanism of pediatric pseudotumor cerebri syndrome: evidence of bioenergetic and hormonal regulation of cerebrospinal fluid dynamics. Pediatr Res. 2015:77:282-9.

37. Salpietro V, Ruggieri M. Pseudotumor cerebri pathophysiology: the likely role of aldosterone. Headache. 2014;54:1229.

38. Khan MU, Khalid H, Salpietro V, Weber KT. Idiopathic intracranial hypertension associated with either primary or secondary aldosteronism. Am J Med Sci. 2013;346:194-8.

39. Narula S, Liu GT, Avery RA, Banwell B, Waldman AT. Elevated cerebrospinal fluid opening pressure in a pediatric demyelinating disease cohort. Pediatr Neurol. 2015 Apr;52:446-9.

40. Voskuhl RR, Palaszynski K. Sex hormones in experimental autoimmune encephalomyelitis: implications for multiple sclerosis. Neuroscientist. 2001;7:258-70

41. Cossburn M, Ingram G, Hirst C, Ben-Shlomo Y, Pickersgill TP, Robertson NP. Age at onset as a determinant of presenting phenotype and initial relapse recovery in multiple sclerosis. Mult Scler. 2012;18:45-54

42. Morley JE, Kaiser FE, Perry HMIII, Patrick P, Morley PM, Stauber PM, Vellas B, Baumgartner RN, Garry PJ. Longitudinal changes in testosterone, luteinizing hormone, and follicle-stimulating hormone in healthy older men. Metabolism. 1997:46:410-3.

43. Gold SM, Voskuhl RR. Estrogen and testosterone therapies in multiple sclerosis. Progr Brain Res. 2009;175:239-51.

44. Whitacre CC. Sex differences in autoimmune disease. Nat Immunol. 2001:2:777-80.

45. Walker $L S$, Abbas AK. The enemy within: keeping selfreactive $T$ cells at bay in the periphery. Nat Rev Immunol. 2002:2:11-9.

46. Hince M, Sakkal S, Vlahos K, Dudakov J, Boyd R, Chidgey A. The role of sex steroids and gonadectomy in the control of thymic involution. Cell Immunol. 2008;252:122-38

47. Bove R, Chitnis T. The role of gender and sex hormones in determining the onset and outcome of multiple sclerosis. Mult Scler. 2014 Apr;20:520-6.

48. Coyle PK. Pregnancy and multiple sclerosis. Neurol Clin. 2012:30:877-88.

49. Sicotte NL, Liva SM, Klutch R, Pfeiffer P, Bouvier S, Odesa S, TC W, Voskuhl RR. Treatment of multiple sclerosis with the pregnancy hormone estriol. Ann Neurol. 2002;52:421-8.

50. Bebo BF Jr, Fyfe-Johnson A, Adlard K, Beam AG, Vandenbark AA, Offner $\mathrm{H}$. Low dose estrogen therapy ameliorates experimental autoimmune encephalomyelitis in two different inbred mouse strains. J Immunol. 2001;166:2080-9.

51. Rubtsova K, Marrack P, Rubtsov AV. Sexual dimorphism in autoimmunity. J Clin Invest. 2015:125:2187-93.

52. Sloka JS, Pryse-Phillips WE, Stefanelli M. The relation between menarche and the age of first symptoms in a multiple sclerosis cohort. Mult Scler. 2006;12:333-9

53. Ramagopalan SV, Valdar W, Criscuoli M, DeLuca GC, Dyment DA, Orton SM, Yee IM, Ebers GC, Sadovnick AD. Age of puberty and the risk of multiple sclerosis: a population based study. Eur J Neurol. 2009:16:342-7.

54. D'Hooghe B, Haentjens MP, Nagels G, D'Hooghe T, De Keyser J. Menarche oral contraceptives, pregnancy and progression of disability in relapsing onset and progressive onset multiple sclerosis. J Neurol. 2012;259:855-61.

55. Chirico V, Lacquaniti A, Salpietro V, Buemi M, Salpietro C, Arrigo T Central precocious puberty: from physiopathological mechanisms to treatment. J Biol Regul Homeost Agents. 2014;28:367-75.

56. Villamor E, Marin C, Mora-Plazas M, Baylin A. Vitamin D deficiency and age at menarche: a prospective study. Am J Clin Nutr. 2011;94:1020-5.

57. Munger KL, Chitnis T, Ascherio A. Body size and risk of MS in two cohorts of US women. Neurology. 2009;73:1543-50.

58. Lee JM, Appugliese D, Kaciroti N, Corwyn RF, Bradley RH, Lumeng JC. Weight status in young girls and the onset of puberty. Pediatrics. 2007;119:e624-30.

59. Davison KK, Susman EJ, Birch LL. Percent body fat at age 5 predicts earlier pubertal development among girls at age 9. Pediatrics. 2003;111:815-21.

60. Winer S, Paltser G, Chan Y, Tsui H, Engleman E, Winer D, Dosch HM. Obesity predisposes to Th17 bias. Eur J Immunol. 2009;39:2629-35.

61. Demerath EW, Towne B, Wisemandle W, Blangero J, Chumlea WC, Siervogel RM. Serum leptin concentration, body composition, and gonadal hormones during puberty. Int J Obes Relat Metab Disord. 1999:23:678-85.

62. Harlan SM, Rahmouni K. PI3K signaling: a key pathway in the control of sympathetic traffic and arterial pressure by leptin. Mol Metab. 2013;2:69-73. 
63. Beretta M, Bauer M, Hirsch E. PI3K signaling in the pathogenesis of obesity: the cause and the cure. Adv Biol Regul. 2015;58:1-15.

64. Ruggieri M, Praticò AD. Mosaic neurocutaneous disorders and their causes. Semin Pediatr Neurol. 2015:22:207-33.

65. Vahidnezhad H, Youssefian L, Uitto J. Molecular genetics of the PI3K-AKTmTOR pathway in Genodermatoses: diagnostic implications and treatment opportunities. J Invest Dermatol. 2015 Sep 24; https://doi.org/10.1038/jid. 2015.331.

66. Lebrun-Julien F, Bachmann L, Norrmén C, Trötzmüller M, Köfeler H, Rüegg MA, Hall MN, Suter U. Balanced mTORC1 activity in oligodendrocytes is required for accurate CNS myelination. J Neurosci. 2014;34:8432-48.

67. Conway RL, Pressman BD, Dobyns WB, Danielpour M, Lee J, Sanchez-Lara PA, Butler MG, Zackai E, Campbell L, Saitta SC, Clericuzio CL, Milunsky JM, Hoyme HE, Shieh J, Moeschler JB, Crandall B, Lauzon JL, Viskochil DH, Harding B, Graham JM Jr. Neuroimaging findings in macrocephaly-capillary malformation: a longitudinal study of 17 patients. Am J Med Genet A. 2007:143A:2981-08.

68. Matarese G, La Cava A, Sanna V, Lord GM, Lechler Rl, Fontana S, Zappacosta S. Balancing susceptibility to infection and autoimmunity: a role for leptin? Trends Immunol. 2002;23:182-7.

69. Matarese G, Carrieri PB, Montella S, De Rosa V, La Cava A. Leptin as a metabolic link to multiple sclerosis. Nat Rev Neurol. 2010;6:455-61.

70. Procaccini C, Pucino V, Mantzoros CS, Matarese G. Leptin in autoimmune diseases. Metabolism. 2015;64:92-104.

71. De Rosa V, Procaccini C, Cali G, Pirozzi G, Fontana S, Zappacosta S, La Cava A, Matarese G. A key role of leptin in the control of regulatory T cell proliferation. Immunity. 2007;26:241-55.

72. Sandyk R, Awerbuch Gl. Multiple sclerosis: the role of the pineal gland in its timing of onset and risk of psychiatric illness. Int J Neurosci. 1993;72:95-106.

73. Lage M, Garcia-Mayor RV, Tomé MA, Cordido F, Valle-Inclan F, Considine RV, Caro JF, Dieguez C, Casanueva FF. Serum leptin levels in women throughout pregnancy and the postpartum period and in women suffering spontaneous abortion. Clin Endocrinol. 1999;50:211-6.

74. Chehab FF. 20 years of leptin: leptin and reproduction: past milestones, present undertakings, and future endeavors. J Endocrinol. 2014;223:T37-48,

\section{Submit your next manuscript to BioMed Central and we will help you at every step:}

- We accept pre-submission inquiries

- Our selector tool helps you to find the most relevant journal

- We provide round the clock customer support

- Convenient online submission

- Thorough peer review

- Inclusion in PubMed and all major indexing services

- Maximum visibility for your research

Submit your manuscript at www.biomedcentral.com/submit

) Biomed Central 